\title{
Revascularización coronaria endovascular en pacientes octogenarios
}

\author{
Endovascular coronary revascularization in octogenarians
}

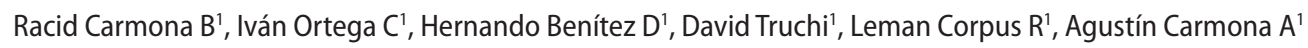

\section{RESUMEN}

Introducción: la cardiopatía isquémica es la principal causa de muerte en la población mundial, los pacientes añosos tienen mayor probabilidad de sufrir enfermedad coronaria. A pesar de las comorbilidades, anatomía coronaria y los cambios fisiológicos asociados a la edad, la revascularización percutánea puede ser realizada con resultados aceptables. Objetivo: mostrar nuestra experiencia en revascularización arterial coronaria, mediante angioplastia transluminal percutánea, en pacientes $(P)$ octogenarios. Material y métodos: se realizó un registro prospectivo consecutivo de pacientes sometidos a estudios coronariográficos entre marzo de 2017 y febrero de 2018, con énfasis en los resultados de aquellos pacientes octogenarios que requirieron tratamiento endovascular (ATCP). Resultados: de un total de 435 pacientes, 56 eran octogenarios, de los cuales 44 tenían enfermedad coronaria severa. La edad promedio fue de $82,4 \pm 2,4$ años, $59 \%$ fue sexo masculino. El estudio coronariográfico se efectuó principalmente por IAM (29,5\%) y Al (54,5\%). El 91\% tenía HTA y el $43 \%$, diabetes tipo II. La ATCP fue electiva en $56,6 \%$, el resto de emergencia, con una tasa de éxito de $94,1 \%$. Conclusión: la ATCP es un procedimiento factible de realizar en este grupo etario con buenos resultados.

\begin{abstract}
Introduction: the ischemic heart disease is the leading cause of death in the word's population. despite comorbidities, coronary anatomy and physiological changes associated with age, the revascularization percutaneous can usually be done with acceptable results. Objective: to show our experience in octogenarian patients who underwent PTCA. Material and method: from a serial prospective registration of patients who underwent coronary angiography studies between march 2017-february 2018, all the octogenarian patients subjected to PTCA were evaluated, with emphasis in the results of the PTCA. Results: of a total of 435 patients, 56 were octogenarian patients, 44 had severe coronary disease. The average age was $82.4 \pm 2.4$ years, $59 \%$ male. They were studied mainly by myocardial infraction (MI) (29.5\%) and unstable angina (54.5\%); $91 \%$ were hypertensive and $43 \%$ had diabetes. The PTCA was elective in $56.6 \%$, the rest were emergency procedures. The PTCA was successful in $94.1 \%$. Conclusion: the PTCA is a feasible procedure to perform in octogenarian patients, with good results.
\end{abstract}

Keywords: coronary artery disease, octogenarians, PTCA.

Palabras claves: enfermedad coronaria, octogenarios, angioplastia coronaria.

Revista Argentina de Cardioangiología Intervencionista 2018;9(4):195-197. DOI: 10.30567/RACI/201804/0195-0197

\section{INTRODUCCIÓN}

La cardiopatía isquémica es la principal causa de muerte en la población mundial, los pacientes añosos tienen mayor probabilidad de sufrir enfermedad coronaria, su cuadro clínico es muy variable, puede presentarse como un síndrome coronario agudo (SCA) (AI, IAM con y sin elevación del segmento ST), y frecuentemente con morfología coronaria adversa y mayores comorbilidades (aterosclerosis, hipertensión arterial (HTA), enfermedad vascular periférica e insuficiencia renal).

Además, los cambios fisiológicos asociados a la edad, tales como disfunción ventricular sistólica, diastólica, rigidez arterial incrementada, y disfunción endotelial, pueden contribuir a un peor pronóstico en pacientes añosos con SCA, a pesar de lo cual, la revascularización percutánea puede usualmente ser realizada con resultados aceptables ${ }^{2-4}$

Debido al crecimiento de la población adulta con una alta prevalencia de la enfermedad coronaria, el tratamiento de la revascularización en esta población debe responder la siguiente pregunta: ¿Es pertinente el tratamiento invasivo frente a un posible tratamiento médico conservador? ${ }^{1}$.

\section{OBJETIVO}

El objetivo de este trabajo es mostrar nuestra experiencia en

1. Servicio de Cardiología Intervencionista, Clínica General del Caribe, Cartagena-Bolívar, Colombia

$\triangle$ Correspondencia:Dr. Racid carmona, racid1981@hotmail.com

Los autores no declaran conflictos de intereses

Recibido: 10/04/2018|Aceptado: 29/11/2018 revascularización arterial coronaria, mediante angioplastia transluminal percutánea, en pacientes $(\mathrm{P})$ octogenarios.

\section{MATERIAL Y MÉTODO}

Se realizo un registro prospectivo consecutivo de pacientes sometidos a estudios coronariográficos entre Marzo de 2017 a febrero de 2018. En total se realizaron 435 cinecoronariografias, de las cuales 56 fueron pacientes octogenarios, en los que se evalúo sus características demográficas y clínicas, con énfasis en los resultados de aquellos que requirieron tratamiento endovascular (ATC).

\section{Definiciones:}

1. Muerte de causa cardiovascular: toda muerte en la que se demuestra una causa cardiovascular o que no es claramente atribuible a una causa no cardiovascular

2. Infarto de miocardio: evento coronario agudo diferente al que motivó la inclusión en el estudio definido por síntomas de isquemia miocárdica y evidencia electrocardiográfica, analítica (biomarcadores de necrosis) o patológica de infarto según la definición de la AHA/ACC.

3. Éxito angiográfico: presencia de lesión residual después de angioplastia con balón de $50 \%$ o menos, o de $30 \%$ o menos con stent, con flujo Timi 2-3 en ambos casos.

4. Angioplastia electiva: es cuando se realiza en un paciente compensado médicamente y sin compromiso hemodinámico al momento del procedimiento.

5. Angioplastia de urgencia: corresponde a cualquiera de las siguientes condiciones: ATC primaria la que es realizada en las primeras $12 \mathrm{~h}$ de un IAM con supradesnivel del ST; ATC de emergencia la realizada en un (P) con SCA que no 


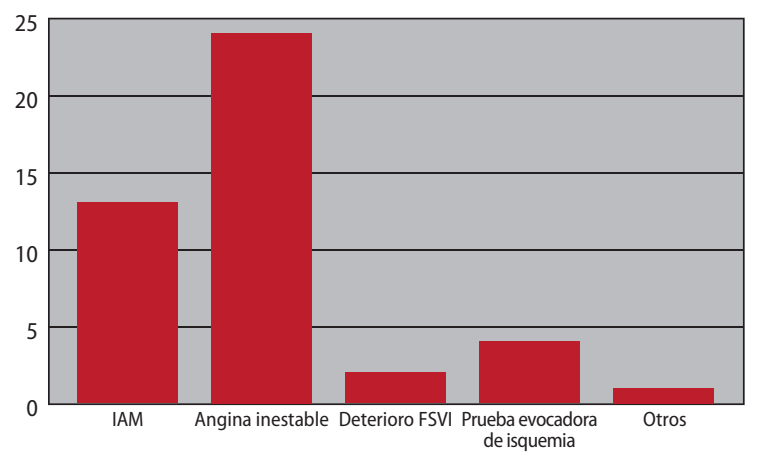

Figura 1.

TABLA 1. Factores de riesgo

\begin{tabular}{|l|c|c|}
\hline & \multicolumn{2}{|c|}{ Pacientes } \\
\hline Edad (media) & 82.4 & $100 \%$ \\
\hline Sexo (M) & 26 & $59 \%$ \\
\hline Sexo (F) & 18 & $41 \%$ \\
\hline HTA & 40 & $91 . \%$ \\
\hline Dislipemia & 32 & $72 \%$ \\
\hline Diabetes mellitus & 19 & $43 \%$ \\
\hline IAM previo & 6 & $13.6 \%$ \\
\hline ATC previa & 3 & $6.8 \%$ \\
\hline CRM previa & 5 & $11.3 \%$ \\
\hline Tabaquismo & 10 & $22.7 \%$ \\
\hline Obesidad & 8 & $18 \%$ \\
\hline
\end{tabular}

TABLA 3. Vasos tratados.

\begin{tabular}{|l|c|}
\hline & $\mathrm{N} \%$ \\
\hline $\mathrm{TCl}$ & $1(3.3 \%)$ \\
\hline Descendente anterior & $16(53.3 \%)$ \\
\hline Ramo diagonal & $9(30 \%)$ \\
\hline Circunfleja & $10(33 \%)$ \\
\hline Ramo marginal & $3(10 \%)$ \\
\hline Coronaria derecha & $15(50 \%)$ \\
\hline Descendente posterior & $7(23.3 \%)$ \\
\hline
\end{tabular}

logra ser compensado medicamente y ATC de salvatajees la efectuada a un $(\mathrm{P})$ con enfermedad coronaria severa, rechazado por cirugía por su compromiso general y comorbilidad. Objetivo primario: combinado de muerte cardiovascular, IAM o ACV no fatal.

Objetivo secundario: tasa de sangrado mayor o menor intra-hospitalario, tasa de nefropatía inducida por contraste.

\section{RESULTADOS}

De un total de $56 \mathrm{P}$ octogenarios sometidos a estudio coronariográfico, en 12 (21\%) se encontraron coronarias sanas y en 44 $(78,5 \%)$ se halló enfermedad coronaria severa, de los cuales 30 $(68,1 \%)$ fueron revascularizados mediante angioplastia coronaria y los $14(31,9 \%)$ restantes fueron derivado a tratamiento medico y/o quirúrgico. La edad promedio de estos fue de $82,4 \pm 2,4$ años (80-90), de los cuales un 59\% fue de sexo masculino. Al analizar los factores de riesgo (Tabla 1) presentes en este grupo, los principales fueron la hipertensión arterial (HTA), dislipidemia (DLP) y diabetes mellitus (DM). En cuanto a comorbilidad (Tabla 2), 26 pacientes (59\%) tenían comorbilidades asociadas. Del total de pacientes, 14 (31,8\%) habían estado previamente hospitalizados por motivos cardiovasculares.

El estudio coronariográfico se efectuó principalmente por cuadros coronarios agudos, 13 P por IAM (29,5\%), 24 por AI (54,5\%), con bajo porcentaje de otros motivos de estudio

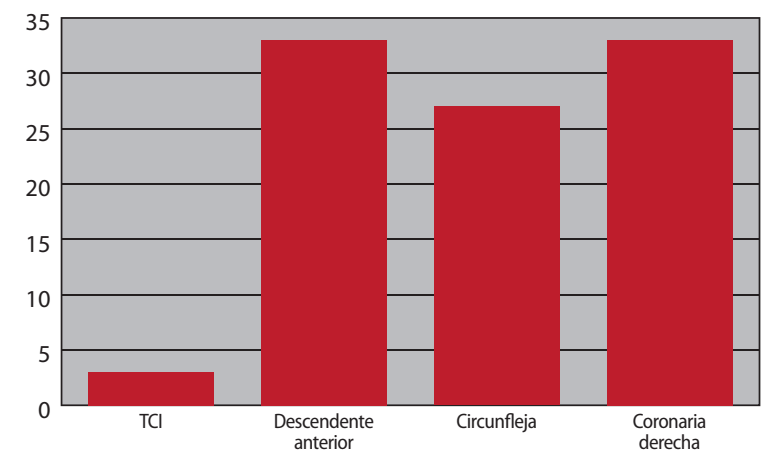

Figura 2 Compromiso coronario.

TABLA 2. Comorbilidades

\begin{tabular}{|l|c|}
\hline & Pacientes \\
\hline Enfermedad pulmonar crónica (EPOC) & $3(10 \%)$ \\
\hline Insuficiencia renal crónica (IRCr) en hemodiálisis (HD) & $2(6.6 \%)$ \\
\hline IRCr sin HD & $4(13.3 \%)$ \\
\hline Enfermedad vascular periférica & $3(10 \%)$ \\
\hline Accidente cerebrovascular (ACV) & $1(3.3 \%)$ \\
\hline Aneurisma aórtico & $1(3.3 \%)$ \\
\hline Enfermedad carotídea & $1(3.3 \%)$ \\
\hline Marcapaso definitivo & $1(3.3 \%)$ \\
\hline Hipotiroidismo & $2(6.6 \%)$ \\
\hline Cáncer & $1(3.3 \%)$ \\
\hline Total & $19(63 \%)$ \\
\hline
\end{tabular}

TABLA 4. ATC electiva vs. urgencia.

\begin{tabular}{|c|c|c|}
\hline Tipo ATC & Éxito & Mortalidad \\
\hline Electiva & $16 / 17(94.1 \%)$ & $0 / 17(0 \%)$ \\
\hline Urgencia & $11 / 13(84.6 \%)$ & $2 / 13(15.3 \%)$ \\
\hline Total & $27(90 \%)$ & $2 / 30(6.6 \%)$ \\
\hline
\end{tabular}

(Figura 1). De este grupo, 39 (88\%) tenían electrocardiograma alterado al momento del ingreso, y solo 4 (9\%) de ellos tenía prueba de isquemia miocárdica realizada al momento del estudio (4 pruebas de esfuerzo). Presentaron lesión de vaso único 13 (29.5\%), dos vasos 9 (20.4\%), tres vasos 16 (36.3\%), tronco más tres vasos 1 (2.2\%), enfermedad coronaria más bypass 5 (11.3\%). El desglose de los vasos comprometidos lo vemos en la Figura 2.

El tratamiento de revascularización con ATC se realizó en 30 pacientes de la siguiente forma: electiva a $17 \mathrm{P}(56,6 \%)$, y a 13P (43,3\%) de urgencia, de ellos 8 fueron ATC primaria $(26,6 \%)$, 3 ATC de emergencia (10\%) y 2 ATC de salvataje $(6,6 \%)$.

En 18 ATC (60\%) se trató un único vaso, en 9 (30\%) dos vasos y en 3 procedimientos (10\%) se procedió a tratar 3 vasos (Tabla 3). El promedio de vasos tratados por cada ATC es de $1,27 \pm 0,52$. Se procede con colocación de stent en 25 intervenciones (83,3\%). Se considera que el $90 \%$ (27 de 30 ) de los procedimientos fue exitoso, un $94,1 \%$ en caso de ATC electiva (Tabla 4) En cuanto al objetivo primario, dos pacientes $(6,6 \%)$ murieron durante su estadía. La mortalidad ocurre solo en el grupo sometido a ATC de urgencia, de ellos 2 estaban cursando un IAM. El desglose es el siguiente: muere el total de los sometidos a ATC primaria (1/8) y $1 \mathrm{P}$ en el que se realizó ATC de emergencia $(1 / 3)$.

En el objetivo secundario se observo que post ATC4 pacientes se complicaron (13,3\%), de los cuales $1(3,3 \%)$ con un hematoma importante en el sitio de punción, manejado exitosamente con compresión localy 3 (10\%) con insuficiencia renal aguda recuperada. 


\section{DISCUSIÓN}

Se estudió un grupo de pacientes octogenarios sometidos a cinecoronariografia haciendo énfasis en aquellos a los que se le realizó ATC, cuyas características se ajustan a las descritas en la literatura. Los pacientes incluidos en el trabajo se estudian fundamentalmente por SCA (IAM y AI suman un 84\%), presentan un contexto de patologías asociadas importante, en el que se incluyen por un lado los factores de riesgo cardiovascular, tales como HTA en un $91 \%$ del grupo, dislipidemia y DM en otro porcentaje, y por otro lado, las comorbilidades asociadas, tales como enfermedad pulmonar o insuficiencia renal. En cuanto a las comorbilidades, un 33,3\% del grupo está presentada fundamentalmente por IRC, enfermedad arterial periférica y aterosclerosis, coincidiendo con las halladas en otros trabajos ${ }^{3,8}$. Solo el 37\% de los pacientes en nuestro grupo estuvo libre de las comorbilidades descritas, es eventualmente explicable por una selección de pacientes, la que estaría condicionada precisamente por la edad de los mismos.

Como se describe en los resultados, la ATC fue exitosa en un $90 \%$ de los casos. Otros autores han demostrado una mayor tasa de éxito, esto probablemente se deba a una mayor experiencia en el manejo de este tipo de pacientes. La evidencia científica demuestra que en el SCA el tratamiento agresivo (revascularización quirúrgica y percutánea), representa un mayor beneficio comparado con el manejo médico ${ }^{9-11}$.

De Luca y cols. ${ }^{5}$ demuestran en su estudio que, a pesar de una mayor mortalidad a largo plazo comparada con pacientes jóvenes ( $\mathrm{HR}=2,17$; IC95\%: 1,97-2,39), la edad no tuvo impacto en el riesgo de re-infarto $(\mathrm{p}=0,36)$, trombosis del stent $(p=0,84)$ ni revascularización del vaso culpable $(p=0,54)^{5,6}$. Por su parte, Fox y cols. ${ }^{7}$, evaluaron el impacto de realizar la revascularización invasiva rutinaria (IR) vs. selectiva (IS) en 5467 pacientes con IAMNST, de los cuales el 48,6\% eran $\geq 65$ años, encontrando que aquellos llevados a IR presentaron menor riesgo de muerte cardiovascular o IAM no fa$\operatorname{tal}(\mathrm{p}=0,002)$.

El beneficio de la terapia invasiva no solo impacta en el SCA, ya que la revascularización ha demostrado ser un método adecuado en pacientes ancianos con enfermedad cardíaca isquémica estable, en donde estudios como el TIME y el
APPROACH demostraron una mejoría sintomática, en la calidad de vida y una reducción en la muerte, el infarto de miocardio y la readmisión por SCA a los 6 meses y a los 4 años, respectivamente ${ }^{12}$.

El estudio «Aftereighty», realizado en pacientes con diagnóstico de IAMNST o angina inestable y edad $\geq 80$ años de edad, demostró que la estrategia invasiva, incluyendo la PCI y el bypass coronario más la terapia médica óptima es superior al tratamiento médico óptimo solo, en pacientes clínicamente estables, sin diferencias en las tasas de complicaciones entre ambos grupos ${ }^{1}$.

En el registro argentino de angioplastia coronaria (RAdAC) ${ }^{13}$ del total de 2919 p, se incluyeron 212 p (7,26\%)mayores de 80 años, de este subgrupo mas de la mitad tenia enfermedad multivaso, el éxito angiografico fue de (97,6\%), con una mortalidad y MACE intrahospitalario de (4,2\% y $6,6 \%$ respectivamente), evidenciándose que la angioplastia coronaria en octogenarios puede ser realizada con una alta tasa de éxito y un bajo riesgo de complicaciones.

No se presentaron complicacionesintra procedimiento en nuestro grupo de pacientes, y solo un porcentaje mínimo presentó complicaciones posprocedimiento que fueron transitorias y resueltas. En la bibliografía se describe que las complicaciones en este grupo etario, no son más frecuentes ni más severas que en grupos de menos edad ${ }^{2,4}$.

La mortalidad global en este trabajo suma un 6,6\%; corresponde a los pacientes cuya ATC fue de urgencia, lo que de cierto modo es esperable y concordante con lo publicado. El evento agudo en el octogenario aumenta significativamente la mortalidad en pacientes sometidos a revascularización percutánea $^{3,8}$.

\section{CONCLUSIÓN}

De acuerdo a los resultados y a la evidencia bibliográfica, encontramos que la angioplastia coronaria en forma electiva se puede realizar satisfactoriamente en este grupo etario de pacientes, con alto éxito angiográfico y sin mayores complicaciones tanto intra- como posprocedimiento. Aquellos pacientes que se someten a revascularización de urgencia tienen una mortalidad substancialmente mayor.

\section{BIBLIOGRAFÍA}

1. CarolinaPemberthy López,CésarCaraballo Cordovez,Simón GalloEcheverri, et al. Tratamiento del adulto mayor con síndrome coronario agudo, Revista Colombiana de Cardiologia, 2016;23 (6): 514-522

2. Héctor UgaldeP, Gonzalo Pizarro J, Ximena Dreyse O, et al. Revista Hospital Clinico Universidad de Chile 2007; 18; 202-207

3. Klein $L W, B l o c k P, B r i n d i s R G$, etal. Percutaneous coronary interventions in octogenariansintheAmerican CollegeofCardiology-NationalCardiovasculardataRegistry:developmentofanomogrampredictiveofin-hospital mortality. Journal American College of Cardiology 2002; 40:394- 402.

4. MajeedF, Kelemen MD. Acute Coronary Syndromes in the Elderly, Clinics in Geriatric Medicine 2007;23:425-40.

5. De Luca G, Dirksen MT, Spaulding C, et al. Impact of Ageon Long-Term Outcome After Primary Angioplasty With Bare-Metal or Drug-Eluting Stent (fromthe DESERT Cooperation). American Journal of Cardiology. 2013;112:181-6.

6. Savonitto S, Morici N, De Servi S. Treatment of Acute Coronary Syndromes in the Elderly and in Patients With Comorbidities. Revista Española de Cardiologia. 2014;67:564-73.

7. zzzzzZleroudi MO, Kleiman NS, Minor ST, et al. Percutaneous transluminal co-

ronary angioplasty in octogenarians. Annalsof Internal Medicine. $1990 \mathrm{Sep}$ 15;113:423-8.

8. Amsterdam EA, Wenger NK, Brindis RG, et al. 2014 AHAVACC Guideline for the Management of Patients with Non-ST-Elevation Acute Coronary Syndromes. Journal American College of Cardiology. 2014;64:e139-228.

9. Alexander KP, Roe MT, Chen AY, et al. Evolution in cardiovascular care for elderIy patients with non-ST-segment elevation acute coronary syndromes: results from the CRUSADE National Quality Improvement Initiative. Journal American College of Cardiology. 2005;46:1479-87.

10. Schoenenberger AW, Radovanovic D, Windecker S, et al. Temporal trends in thetreatment and outcomes of elderly patients with acute coronary syndrome. European Heart Journal. 2016;37:1304-11.

11. McCune C, McKavanagh P, Menown IB. A Review of Current Diagnosis, Investigation, and Management of Acute Coronary Syndromes in Elderly Patients. Cardiology and Therapy journal. 2015:4:95-116

12. Rubilar B, Martin R, Coroleu S, et al. Resultados intrahospitalarios de la angioplastia coronaria en octogenarios. Sub-estudio del Registro Argentino de Angioplastia Coronaria (RAdAC). Revista Argentina de Cardioangiología Intervencionista 2015;6(4):180-186 